A case-controlled study of Dientamoeba fragilis

\title{
infections in children
}

4

G. R. BANIK ${ }^{1,2,3}$, J. L. N. BARRATT ${ }^{1,2,3}$, D. MARRIOTT ${ }^{1,3}$, J. HARKNESS ${ }^{1,3}$,

6

J. T. ELLIS ${ }^{2,3}$ and D. STARK ${ }^{1,3 *}$

7

$8 \quad{ }^{1}$ Division of Microbiology, SydPath, St. Vincent's Hospital, Darlinghurst, Australia

$9 \quad{ }^{2}$ University of Technology Sydney, i3 Institute, Broadway, Australia

$10{ }^{3}$ University of Technology Sydney, School of Medical and Molecular Biosciences, Broadway,

11 Australia

12

Running title: Dientamoeba fragilis infections in children

14

15

16 *Corresponding author: Department of Microbiology, St.Vincent's Hospital, Darlinghurst

2010, NSW, Australia. Tel: +61 28382 9196. Fax: +61 283822989 E-mail:

dstark@stvincents.com.au

19

20

21

22

23

24

25 


\section{SUMMARY}

Dientamoeba fragilis is a pathogenic protozoan parasite that is implicated as a cause of human diarrhea. A case-controlled study was conducted to determine the clinical signs associated with $D$. fragilis infection in children presenting to a Sydney Hospital. Treatment options are also discussed. Stool specimens were collected from children aged 15 years or younger and analysed for the presence of $D$. fragilis. A total of 41 children were included in the study along with a control group. Laboratory diagnosis was performed by microscopy of permanently stained fixed faecal smears and by real-time PCR. Gastrointestinal symptoms were present in 40/41 (98\%) of these children with dientamoebiasis, with diarrhea (71\%) and abdominal pain (29\%) the most common clinical signs. Chronic gastrointestinal symptoms were present in $2 \%$ of cases. The most common antimicrobial used for treatment was metronidazole $(\mathrm{n}=41)$, with complete resolution of symptoms and clearance of parasite occurring in $85 \%$ of cases. A treatment failure rate occurred in $15 \%$ of those treated with metronidazole. Follow-up treatment comprising of an additional course of metronidazole or iodoquinol was needed in order to achieve complete resolution of infection and symptoms in this group. This study demonstrates the pathogenic potential of $D$. fragilis in children and as such it is recommended that all laboratories must routinely test for this organism and treat if detected.

Key words: Dientamoeba fragilis; children; gastrointestinal symptoms; metronidazole, diarrhoea. 
53

54

55

56

57

58

59

60

61

62

63

64

65

66

67

68

69

70

\section{INTRODUCTION}

Dientamoeba fragilis is a trichomonad-like protozoan parasite with a worldwide distribution commonly found in the gastrointestinal tract of humans (Stark et al. 2006; Cepicka et al. 2010; Stark et al. 2010a,b; Barratt et al. 2011a). Despite widespread belief to the contrary, numerous reports document that $D$. fragilis is a common cause of gastrointestinal disease in both developed and developing regions of the world and has the propensity to exist as a chronic infection with associated clinical signs of disease (Grendon et al. 1991; Grendon et al. 1995; Dickinson et al. 2002; Norberg et al. 2003; Stark et al. 2006; Stark et al. 2010b; Barratt et al. 2011a). It is found in all patient groups studied so far including the general population, travellers and HIV infected individuals (Stark et al. 2005; Stark et al. 2007a; Stark et al. 2007b; Barratt et al. 2011a).

Several studies have reported that children are susceptible to infection with $D$. fragilis and present with clinical symptoms at higher rates than adults (Preiss et al. 1991; Ayadi \& Bahri, 1999; Crotti et al. 2005). Consequently, D. fragilis should be considered in the differential diagnosis of gastrointestinal infections in children (Spencer et al. 1979; Keystone et al. 1984; Preiss et al. 1990; Stark et al. 2009) but it is generally ignored as a cause of disease. Clinical symptoms reported include acute and chronic diarrhea, lower abdominal pain, nausea, flatulence and constipation ( Spencer et al. 1983; Cuffari et al. 1998). Transmission of $D$. fragilis is believed to be via the fecal-oral route, but the mechanism and whether it includes a helminth or a cyst-like stage, are still unclear (Stark et al. 2006; Barratt et al. 2011b).

Here we review existing knowledge on dientamoebiasis in children. A case-controlled study was also conducted to document the extent of $D$. fragilis infections in children presenting to a major Sydney Hospital. 
80

\section{MATERIALS AND METHODS}

The study was performed at St. Vincent's Hospital, Sydney. Laboratory and clinical records of D. fragilis infected children were collected from August 2004 to July 2010. The following criteria were used for inclusion into this study: children were aged 15 years or less, full clinical history, confirmed laboratory diagnosis of $D$. fragilis as the sole pathogen, treatment history, follow up stool samples were analysed to evaluate treatment regimes, clinical follow up and bacteriological cultures for enteric pathogens and virological screening for the presence of rotavirus and enteric adenoviruses was performed. A total of 41 children were included in the study along with a control group. A control group of children (established using the same criteria) free from infection of $D$. fragilis and confirmed by PCR (as described below), were included in the study. Where possible control subjects were age and sex matched with $D$. fragilis infected children. However, these were not possible for five children. In the majority of these cases $(90 \%, 37 / 41)$ only a single stool sample was collected and analysed.

\section{Microbiological analysis}

Laboratory diagnosis was performed by microscopy of permanently stained fixed faecal smears and by real-time PCR as previously described (Stark et al. 2010a). Bacterial cultures were performed using standard microbiological techniques to rule out the following infections; Salmonella spp., Shigella spp., Campylobacter spp., Yersinia enterocolitica, Vibrio spp., Plesiomonas spp., Aeromonas and Clostridium difficile. Virology testing was performed by an immunochromatographic screening test (Adeno/Rota STAT-PAK; Chembio Diagnostic Systems Inc., Sydney) for the detection of adenovirus and rotavirus antigen in faeces according to the manufacturer's recommendations. 
104

105 Statistical methods

106 Differences between means of gastrointestinal symptoms of control subjects and $D$. fragilis 107 infected cases were evaluated by using a Chi-Square $\left(\chi^{2}\right)$ Test of Association.

108 
110 A total of 41 children were identified from laboratory and hospital records as meeting the

111 inclusion criteria for the study. All 41 children had laboratory confirmed $D$. fragilis infection

112 along with clinical notes detailing symptoms, antimicrobial treatment regime and follow up

113 stool samples to check for parasite clearance following treatment. The study group ranged in

114 age from 1 to 15 years with $88 \%(n=36)$ less than 10 years of age (Fig. 1$)$. None of the

115 children were immunosuppressed. No bacterial pathogens were isolated from the stools of the

11641 D. fragilis infected children. All children's stools were also negative for enteric

117 adenoviruses and rotaviruses. The control group comprised of 41 children, in which no $D$.

118 fragilis was detected by microscopy and PCR. No bacterial or viral pathogens were detected 119 in this group.

120 Initially, 47 D. fragilis infected children were identified from records; among them 121 ten were infected with other enteric parasites; four with Blastocystis hominis, two with

122 Cryptosporidium spp. and B. hominis, two with Endolimax nana, one with Entamoeba coli, 123 and one with Enteromonas hominis. As Cryptosporidium spp. is considered pathogenic and 124 B. hominis is potentially pathogenic and capable of causing gastrointestinal symptoms 125 (Yakoob et al. 2010), these six children were excluded from the data dealing with symptoms 126 and treatment.

Ninety-eight percent (40/41) of the children with $D$. fragilis infection presented with at least one or more gastrointestinal symptoms. Diarrhea (71\%) was found to be the most common symptom followed by abdominal pain (29\%) (Table 1). Persistent or chronic 130 infection was reported in one child. children with dientamoebiasis. Other enteric protozoa were present in 2\% (1/41) of control 
133 children. Originally, 44 control patients were identified, however three were removed

134 because they harbored $B$. hominis and had symptoms of diarrhea.

135 A total of 41 children were treated with metronidazole and 35/41 (85\%) reported

136 complete resolution of symptoms. Follow up stool samples collected between 1-4 weeks

137 following treatment were collected from these children and all showed clearance of the

138 parasite. However in six children, gastrointestinal symptoms did not resolve following initial

139 metronidazole treatment. Four of those children were subsequently treated with a repeat

140 course of metronidazole for either 10 or 14 days and the other two children were treated with

141 iodoquinol. Following this additional antimicrobial therapy the children reported improved

142 clinical symptoms and follow up stool samples collected two weeks post treatment were

143 negative for $D$. fragilis. 


\section{DISCUSSION}

This study, conducted over a 6 year period, highlights the association of $D$. fragilis with clinical signs of disease: $98 \%$ of the children infected with $D$. fragilis studied presented with gastrointestinal symptoms. Diarrhea was found in 29/41 (71\%) of the children and abdominal pain in 12/41 (29\%). The control group represents a group of symptomatic children free of $D$. fragilis infection. Diarrhea was, however, more common in children with $D$. fragilis infection compared to the control group.

There are other reports from various parts of the world that also describe an association between $D$. fragilis infection in children and various clinical symptoms, most commonly diarrhea and abdominal pain. A large study comprising of over 43,029 children first reported a correlation between $D$. fragilis infection and symptoms of diarrhea, abdominal pain and loose stools (Yang \& Scholten, 1977). In that study, chronic infections were found in $2 \%$ of children. Spencer et al. (1983) reported a study from 104 children, in which diarrhea and abdominal pain were the most common symptoms in those with $D$. fragilis infection. Preiss et al. (1990) reported that among 102 children, seven had acute diarrhea, 39 had relapsing diarrhea, seven had bloody stools and 29 had abdominal pain. A retrospective study of 87 Swedish children diagnosed with $D$. fragilis found the majority of children had symptoms of diarrhea, abdominal pain and flatus (Norberg et al. 2003). Previous reports have also highlighted the propensity of the parasite to cause prolonged infection (Stark et al. 2005; Crotti \& D'Annibale, 2007) with chronic infections reported in the literature to last as long as 2 years (Wenrich, 1944).

Dientamoeba fragilis is a commonly encountered enteric protozoan parasite in children that should be considered in any differential diagnosis of gastrointestinal disease. Several studies have shown $D$. fragilis to be more prevalent than Giardia intestinalis in paediatric populations (Preiss et al. 1990; Crotti \& D'Annibale, 2007; Rayan et al. 2007). 
172 One serological study carried out in children reported a D. fragilis seroprevalence of 91\%

173 (Chan et al. 1996). When compared to the seroprevalence of Giardia and Cryptosporidium 174 this study suggests childhood contact with Dientamoeba is common (Chan et al. 1996). Yang 175 and Scholten (1977) found D. fragilis in $4.2 \%$ of individuals who submitted stools for 176 parasitological examination during 1970-1974 in Ontario, Canada. Infections were found to 177 be more common in children, with nearly half of the infections occurring in individuals under 17820 years old (Yang \& Scholten, 1977). A recent study from the Netherlands found that $D$. 179 fragilis infection was most common in children aged between 5-14 years (de Wit et al. 2001). 180 Interestingly, an association was observed between $D$. fragilis infection and carriage of other 181 enteric protozoa normally transmitted via the fecal-oral route. This association has been 182 previously observed (Stark et al. 2010b) and suggests that transmission of $D$. fragilis also occurs in the same way (by the faecal oral route). There was no evidence for the presence of helminths in this study, which have previously been suggested to a host for $D$. fragilis (Stark et al. 2006). Recent studies also suggest that helminths such as Enterobius vermicularis appear to play no role in transmission of D. fragilis (Stark et al. 2010b, Barratt et al. 2011b). Antimicrobial treatment most commonly used for treatment of $D$. fragilis infection in children includes metronidazole and idoquinol (diidohydroxyquin) (Stark et al. 2010b). Therapy with metronidazole was effective for most of the children in this study. Forty one children were administered the drug, with the duration of treatment varying from 5-10 days. $85 \%$ of the children treated with metronidazole (35/41) resulted in the clearance of $D$. fragilis

192 as determined by analyses of follow up stools and complete resolution of gastrointestinal symptoms. However 6/41 (15\%) children who underwent metronidazole treatment failed to clear the infection parasitologically or clinically. There was no correlation between the dose received, the duration of treatment and treatment failure associated with metronidazole use.

196 These six children underwent further treatment, four were put on a repeat course of 
197 metronidazole for 2 weeks duration and the other two children were treated with iodoquinol.

198 On follow up examination all children reported marked clinical improvement and clearance

199 of parasite from stool samples. Iodoquinol has been widely used to treat $D$. fragilis infections

200 (Butler, 1996). In a recent study, 27/33 children were successfully treated with clioquinol, a

201 member of the same drug family as iodoquinol (Bosman et al. 2004).

202 There are varying reports of the efficacy of metronidazole treatment for $D$. fragilis

203 infections in children. Spencer et al. (1979) reported that therapy with metronidazole was

204 effective in 35 children (Spencer et al. 1979). Similarly, in New Zealand, metronidazole was

205 successfully used in the treatment of dientamoebiasis in three children (Oxner et al. 1987).

206 Preiss et al. (1990) studied 123 paediatric children with D. fragilis infections (Preiss et al.

207 1990). They found metronidazole to be effective with $70 \%$ of children eliminating the

208 parasite and symptoms after one treatment. A second treatment was required for 21 other

209 children with another drug. Ten children were treated a third time in order to eliminate $D$.

210 fragilis and accompanying abdominal complaints. They recommended a 10-day treatment

211 with metronidazole for $D$. fragilis infections. Cuffari et al. (1998) showed that metronidazole

212 was effective in treatment of five children (Cuffari et al. 1998). A study from Sweden

213 included 32 children whom were treated with metronidazole. The drug was given at various

214 doses for various lengths of time, and they found that only four children responded to the

215 metronidazole treatment (Norberg et al. 2003). Recently it was also documented that two

216 children presented with $D$. fragilis infection over prolonged periods (Stark et al. 2009). In

217 that study, metronidazole was used initially, but subsequently treatment was done with

218 paramomycin for 10 days to effect clearance of the parasite. At this point in time, it is not

219 clear why some cases of dientamoebiasis do not respond to metronidazole treatment; such

220 observations may be the result of metronidazole resistance or failure to comply with

221 medication (Stark et al. 2010b). 
In conclusion, this study serves to highlight the fact that children infected with $D$.

223 fragilis typically have diarrhea. Studies such as those reported here, and elsewhere, strongly

224 implicate $D$. fragilis as a common cause of gastrointestinal disease in children and other 225 patient populations (Stark et al. 2006; Stark et al. 2010b; Barratt et al. 2011a). No D. fragilis 226 was detected in the control group. We therefore recommend that all laboratories must

227 routinely test for $D$. fragilis as treatment which eliminates the parasite usually results in the 228 resolution of symptoms. It is essential a correct clinical and laboratory diagnosis is made so 229 treatment can be initiated.

230

231

ACKNOWLEDGMENTS

232 We acknowledge the help of the staff at St Vincent's Hospital Microbiology Department in 233 the collection and processing of stool samples positive for $D$. fragilis. Part of this work was 234 presented at the "XIIth International Congress of Parasitology (ICOPA 2010)", Melbourne, 235 Australia (16 ${ }^{\text {th }}$ of August, 2010). This research was supported by a grant from the St. 236 Vincent's Hospital, Darlinghurst, Australia, University of Technology, Sydney and Australian 237 Research Council. Ms S. Fletcher provided advice on statistics. 
REFERENCES

Ayadi, A. and Bahri, I. (1999). Dientamoeba fragilis: pathogenic flagellate? Bulletin de la Société de Pathologie Exotique 92, 299-301.

Barratt, J.L.N., Harkness, J., Marriott, D., Ellis, J.T. and Stark, D. (2011a) A review of Dientamoeba fragilis carriage in man: several reasons why this organism should be considered in the diagnosis of gastrointestinal illness. Gut Microbes 2:1, 1-10.

Barratt, J.L.N., Harkness, J., Marriott, D., Ellis, J.T. and Stark, D. (2011b) The ambiguous life of Dientamoeba fragilis: the need to investigate current hypotheses on transmission. Parasitology, in press.

Bosman, D. K., Benninga, M. A., van De Berg, P., Kooijman, G. C. and van Gool, T. (2004). Dientamoeba fragilis: possibly an important cause of persistent abdominal pain in children. Nederlands Tijdschrift Voor Geneeskunde 148, 575-579.

Butler, W. P. (1996). Dientamoeba fragilis. An unusual intestinal pathogen. Digestive Diseases and Sciences 41, 1811-1813.

Cepicka, I., Hampl, V. and Kulda, J. (2010). Critical taxonomic revision of Parabasalids with description of one new genus and three new species. Protist 161, 400-433.

Chan, F., Stewart, N., Guan, M., Robb, I., Fuite, L., Chan, I., Diaz-Mitoma, F., King, J., MacDonald, N. and Mackenzie, A. (1996). Prevalence of Dientamoeba fragilis antibodies in children and recognition of a $39 \mathrm{kDa}$ immunodominant protein antigen of the organism. European Journal of Clinical Microbiology \& Infectious Diseases 15, 950-954.

Crotti, D. and D'annibale, M. L. (2007). Intestinal infections caused by Dientamoeba fragilis and Giardia duodenalis in our experience. Recenti Progressi in Medicina 98, 361-366.

Crotti, D., D'annibale, M. L., Fonzo, G., Lalle, M., Caccio, S. M. and Pozio, E. (2005). Dientamoeba fragilis is more prevalent than Giardia duodenalis in children and adults attending a day care centre in Central Italy. Parasite 12, 165-170.

Cuffari, C., Oligny, L. and Seidman, E. G. (1998). Dientamoeba fragilis masquerading as allergic colitis. Journal of Pediatric Gastroenterology and Nutrition 26, 16-20.

De Wit, M. A., Koopmans, M. P., Kortbeek, L. M., van Leeuwen, N. J., Vinjé , J. and van Duynhoven., Y. T. (2001). Etiology of gastroenteritis in sentinel general practices in the netherlands. Clinical Infectious Diseases 33, 280-288.

Dickinson, E. C., Cohen, M. A. and Schlenker, M. K. (2002). Dientamoeba fragilis: a significant pathogen. The American Journal of Emergency Medicine 20, 62-63.

Grendon, J. H., Digiacomo, R. F. and Frost, F. J. (1991). Dientamoeba fragilis detection methods and prevalence: a survey of state public health laboratories. Public Health Reports 106, 322-325. 
Grendon, J. H., Digiacomo, R. F. and Frost, F. J. (1995). Descriptive features of Dientamoeba fragilis infections. The Journal of Tropical Medicine and Hygiene $\mathbf{9 8 ,}$ 309-315.

Keystone, J. S., Yang, J., Grisdale, D., Harrington, M., Pillon, L. and Andreychuk, R. (1984). Intestinal parasites in metropolitan Toronto day-care centres. Canadian Medical Association Journal 131, 733-735.

Norberg, A., Nord, C. E. and Evengård, B. (2003). Dientamoeba fragilis--a protozoal infection which may cause severe bowel distress. Clinical Microbiology and Infection 9, 65-68.

Oxner, R. B., Paltridge, G. P., Chapman, B. A., Cook, H. B. and Sheppard, P. F. (1987). Dientamoeba fragilis: a bowel pathogen? The New Zealand Medical Journal 100, 6465.

Preiss, U., Ockert, G., Broemme, S. and Otto, A. (1991). On the clinical importance of Dientamoeba fragilis infections in childhood. Journal of Hygiene, Epidemiology, Microbiology, and Immunology 35, 27-34.

Preiss, U., Ockert, G., Brömme, S., and Otto, A. (1990). Dientamoeba fragilis infection, a cause of gastrointestinal symptoms in childhood. Klinische Pädiatrie 202, 120-123.

Rayan, H. Z., Ismail, O. A. and El Gayar, E. K. (2007). Prevalence and clinical features of Dientamoeba fragilis infections in patients suspected to have intestinal parasitic infection. Journal of the Egyptian Society of Parasitology 37, 599-608.

Spencer, M. J., Garcia, L. S. and Chapin, M. R. (1979). Dientamoeba fragilis. An intestinal pathogen in children? American Journal of Diseases of Children 133, 390393.

Spencer, M. J., Millet, V. E., Garcia, L. S., Rhee, L. and Masterson, L. (1983). Parasitic infections in a pediatric population. Pediatric Infectious Diseases 2, 110-113.

Stark, D., Beebe, N., Marriott, D., Ellis, J. and Harkness, J. (2005). Prospective study of the prevalence, genotyping, and clinical relevance of Dientamoeba fragilis infections in an Australian population. Journal of Clinical Microbiology 43, 2718-2723.

Stark, D. J., Beebe, N., Marriott, D., Ellis, J. T. and Harkness, J. (2006).

Dientamoebiasis: clinical importance and recent advances. Trends in Parasitology 22, 92-96.

Stark, D., Beebe, N., Marriott, D., Ellis, J. and Harkness, J. (2007a). Dientamoeba fragilis as a cause of travelers' diarrhea: report of seven cases. Journal of Travel Medicine 14, 72-73.

Stark, D., Fotedar, R., Van Hal, S., Beebe, N., Marriott, D., Ellis, J. T. and Harkness, J. (2007b). Prevalence of enteric protozoa in human immunodeficiency virus (HIV)positive and HIV-negative men who have sex with men from sydney, australia. The American Journal of Tropical Medicine and Hygiene 76, 549-552. 
Stark, D., Barratt, J., Ellis, J., Harkness, J. and Marriott, D. (2009). 'Repeated Dientamoeba fragilis infections: a case report of two families from Sydney, Australia', Infectious Disease Reports 1, 7-9.

Stark, D., Barratt, J., Roberts, T., Marriott, D., Harkness, J. and Ellis, J. (2010a). Comparison of microscopy, two xenic culture techniques, conventional and real-time PCR for the detection of Dientamoeba fragilis in clinical stool samples. European Journal of Clinical Microbiology \& Infectious Diseases 29, 411-416.

Stark, D., Barratt, J., Roberts, T., Marriott, D., Harkness, J. and Ellis, J. (2010b). A review of the clinical presentation of dientamoebiasis. The American Journal of Tropical Medicine and Hygiene 82, 614-619.

Wenrich, D. H. (1944). Studies on Dientamoeba fragilis (protozoa). IV. Further observations, with an outline of present-day knowledge of this species. The Journal of Parasitology 30, 322-338.

Yakoob, J., Jafri, W., Beg, M. A., Abbas, Z., Naz, S., Islam, M. and Khan, R. (2010). Blastocystis hominis and Dientamoeba fragilis in patients fulfilling irritable bowel syndrome criteria. Parasitology Research 107, 679-684.

Yang, J. and Scholten, T. (1977). Dientamoeba fragilis: a review with notes on its epidemiology, pathogenicity, mode of transmission, and diagnosis. The American Journal of Tropical Medicine and Hygiene 26, 16-22. 


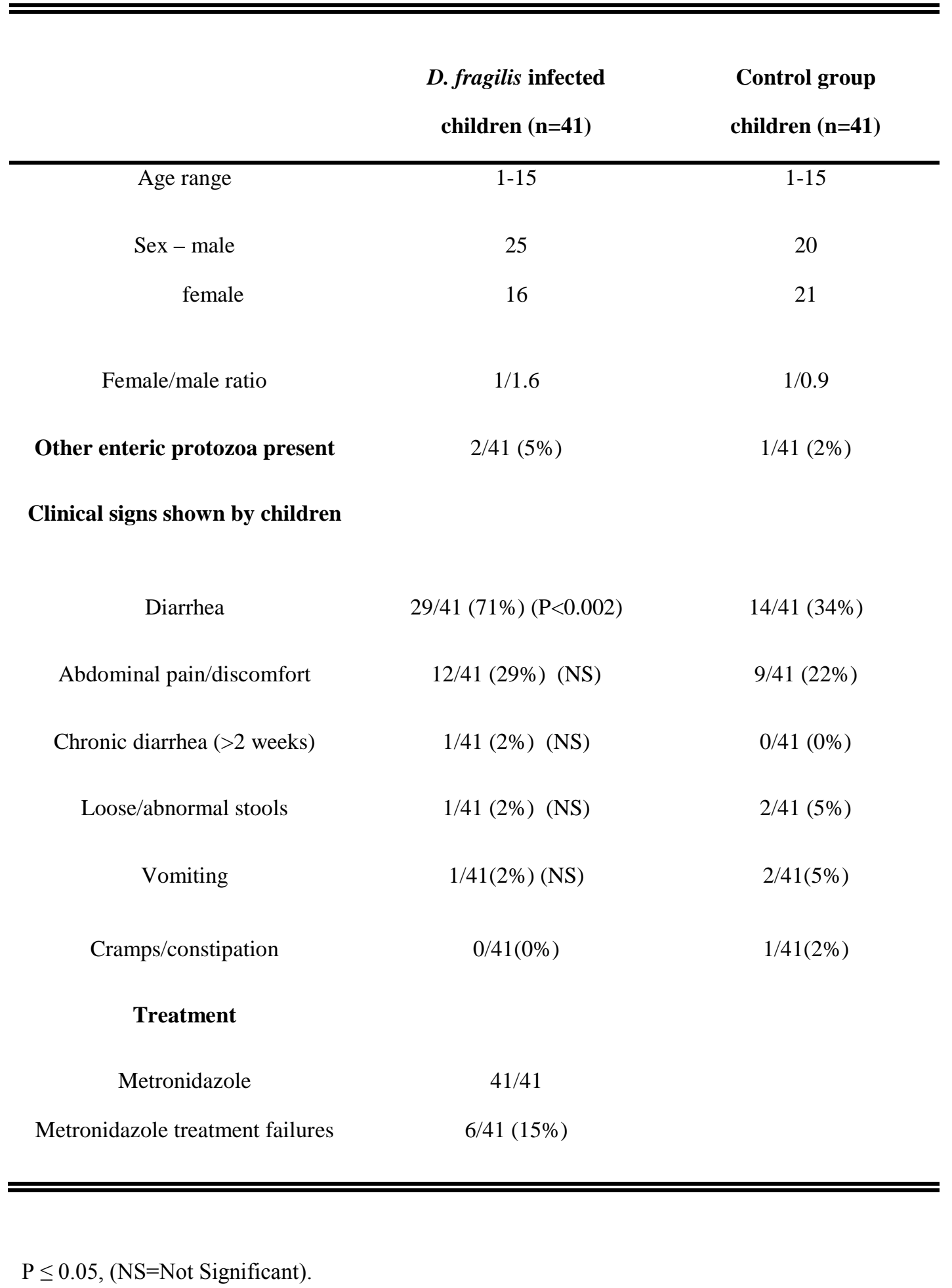


357 LEGENDS TO FIGURE

358 Figure 1. Age of children with dientamoebiasis, (M= Male; F=Female).

359

360

361

362

363

364

365

366

367

368

369

370

371

372

373

374

375

376

377

378

379

380

381

382

383

384

385

386

387

388

389

390

391

392

393

394 


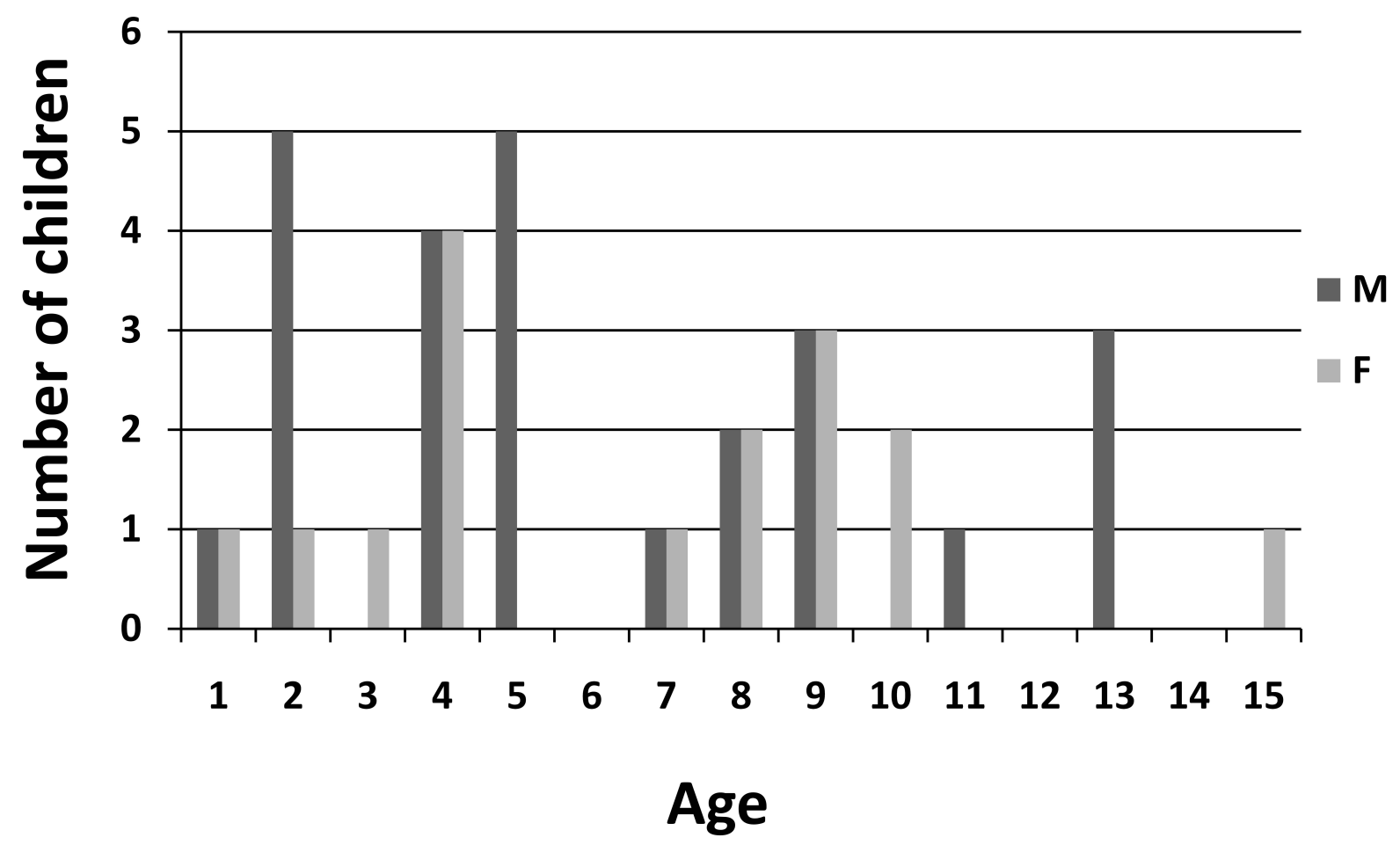

